\title{
DESEMPENHO DE BANANEIRAS CONSORCIADAS COM LEGUMINOSAS HERBÁCEAS PERENES
}

\author{
Banana plant performance intercropping with perennial herbaceous legumes \\ Adriano Perin ${ }^{1}$, José Guilherme Marinho Guerra², José Antonio Azevedo Espindola ${ }^{2}$ \\ Marcelo Grandi Teixeira ${ }^{2}$, Rubens Nei Briançon Busquet ${ }^{3}$
}

\begin{abstract}
RESUMO
O emprego de plantas de cobertura em consórcio com bananeiras pode ser uma estratégia de manejo, possibilitando aumentos de produtividade associados à otimização de processos biológicos e maior estabilidade do sistema produtivo. Neste trabalho, objetivo-se avaliar o efeito da cobertura viva, formada por leguminosas herbáceas perenes sobre a produção de bananeira cultivar Nanicão. Os tratamentos foram: amendoim forrageiro (Arachis pintoi Krap. \& Greg), cudzu tropical (Pueraria phaseoloides Benth.), siratro (Macroptilium atropurpureum Urb.), vegetação espontânea (dominada por Panicum maximum Jacq.) e vegetação espontânea $+\mathrm{N}$-fertilizante. Foi avaliado o desenvolvimento vegetativo das bananeiras entre abril/1999 e julho/2000 e os atributos de produtividade. $\mathrm{O}$ peso do cacho e da penca foram positivamente influenciados pelo siratro e cudzu tropical empregados como coberturas vivas, quando comparados aos demais tratamentos. Todas as leguminosas proporcionaram maior crescimento das bananeiras (notadamente a partir do $6^{\circ}$ mês), maior número de folhas emitidas e maior proporção de cachos colhidos, em relação aos tratamentos com vegetação espontânea (com e sem $\mathrm{N}$-fertilizante). As leguminosas siratro e cudzu tropical promoveram condições adequadas ao desenvolvimento das bananeiras, acarretando ganhos de produtividade e eliminação da adubação nitrogenada no bananal. O potencial benéfico das leguminosas cudzu tropical e siratro como coberturas vivas capazes de proporcionar aumentos na produtividade de banana, qualifica essas espécies como alternativa promissora para a fertilidade do solo e nutrição das bananeiras.
\end{abstract}

Termos para indexação: Musa spp., plantas de cobertura, adubação nitrogenada, adubação verde.

\begin{abstract}
The use of coverage plants in cover cropping with bananas can be a management strategy, increasing in productivity associated with the optimization of biological processes and greater stability of production system. The objective of this work was to evaluate the effect of live coverage by herbaceous perennial legume on the yield of banana cultivar Nanicão. The treatments were: forage groundnut (Arachis pintoi Krap. \& Greg), tropical cudzu (Pueraria phaseoloides Benth.), siratro (Macroptilium atropurpureum Urb.), spontaneous vegetation (mainly Panicum maximum Jacq.) and spontaneous vegetation $+\mathrm{N}$-fertilizer. Banana plant vegetative development and yield attributes between April/1999 and August/2000 were assessed. In comparison to other treatments, bunch and hand weight were more positively influenced by tropical cudzu and siratro legumes employed as live coverage. All legumes promoted higher vegetative growth for banana plants (notably from the 6th month on), a greater number of leaves, and a higher proportion of harvested bunches in relation to spontaneous vegetation (with and without $\mathrm{N}$-fertilizer). Siratro and tropical cudzu legumes promoted adequate conditions for the development of the banana plants, generating yield gains, and the elimination of the need of nitrogenous fertilization in the banana plantation. The beneficial potential of tropical cudzu and siratro legumes as live coverage intercropping with banana plants qualify these species as promising alternatives for soil fertility and banana nutrition.
\end{abstract}

Index terms: Musa spp., cover crops, nitrogen fertilizer, green manure.

(Recebido em 31 de julho de 2008 e aprovado em 3 de novembro de 2008)

\section{INTRODUÇÃO}

A banana é um alimento altamente energético com elevado conteúdo de carboidratos facilmente assimiláveis. Apresenta boa aceitação pelos consumidores, sendo indicada como fonte de proteínas e minerais (Medina et al., 1995). Essa cultura é difundida em praticamente todos os municípios brasileiros, o que torna o Brasil o $3^{\circ}$ maior produtor e consumidor mundial (IBGE, 2002). No estado do Rio de Janeiro, a bananeira assume grande importância econômica e social, visto representar o $2^{\circ}$ maior PIB agrícola do estado (IBGE, 2002). Geralmente, é cultivada em áreas de relevo acidentado, o que exige adoção de práticas de conservação do solo e esse manejo não tem recebido a devida atenção.

${ }^{1}$ Prof, Doutor em Fitotecnia, Instituto Federal Goiano - Campus Rio Verde, Rod. Sul Goiana, km 01 - Cx. P. 66 - $75.901-970$ - Rio Verde, GO perinrj@yahoo.com.br

${ }^{2}$ Pesquisador, Doutor em Agronomia: Ciência do Solo, Embrapa Agrobiologia, BR 465, Km 07 - 23851-970 - Seropédica, RJ - gmguerra@cnpab.embrapa.br ${ }^{3}$ Prof, Doutor em Fitotecnia, Universidade Federal Rural do Rio de Janeiro, Departamento de Fitotecnia, BR 465, km 07 - $23890-000$ - Seropédica, RJ rbusquet@ufrrj.br 
Recentemente, vem sendo avaliado o emprego de plantas de cobertura como estratégia de consórcio com bananeiras. Dentre as espécies estudadas, a ênfase tem sido para as leguminosas herbáceas, de ciclo perene e uso forrageiro, visto permanecerem por vários anos na área. No entanto, é importante a seleção de espécies mais adequadas para cobertura de solo, de forma a garantir o sucesso dessa prática. Borges et al. (1997) apontam diversas características desejáveis a uma planta de cobertura em consórcio com bananeiras: produção de sementes viáveis, boa adaptação às condições edafoclimáticas, facilidade de manejo, resistência a pragas e doenças, competição com ervas espontâneas, tolerância ao sombreamento, e compatibilidade com a bananeira. No uso de plantas de cobertura do solo, mostra-se uma estratégia de manejo para os agroecossistemas, possibilitando aumentos de produtividade associados à otimização de processos biológicos (Espindola et al., 2006b). Entre as vantagens trazidas por essa prática, podem ser destacados a proteção do solo contra agentes climáticos causadores de erosão (Derpsch et al., 1991), o controle de plantas de ocorrência espontânea (Espindola et al., 2000) e o aumento da disponibilidade de nutrientes ligados à matéria orgânica do solo (Canellas et al., 2004).

As leguminosas herbáceas perenes, além de protegerem o solo e competirem com as ervas espontâneas, mantêm ou elevam o teor de matéria orgânica, mobilizam nutrientes de camadas profundas, fixam nitrogênio atmosférico e favorecem a atividade biológica (Wiles et al., 1989; Duda et al., 2003; Perin et al., 2003). Tais benefícios podem proporcionar maior desempenho das culturas de interesse (Espindola et al., 2000) e contribuir para maior estabilidade do sistema produtivo.

$\mathrm{O}$ nitrogênio $(\mathrm{N})$ é um nutriente essencial para o desenvolvimento da bananeira. No entanto, a adubação nitrogenada, em algumas situações, onera os custos de produção desta fruteira. Por isso, o uso de leguminosas herbáceas perenes consorciadas às bananeiras tem sido objeto de investigação, visto que essas plantas fixam carbono e $\mathrm{N}$ atmosféricos que serão disponibilizados para o sistema solo-planta. Contudo, pouco ainda se conhece sobre o comportamento agronômico das leguminosas utilizadas para tal fim, e seus efeitos no desempenho das fruteiras em consórcio.

Nesse trabalho, objetivou-se avaliar o efeito da cobertura viva formada por leguminosas herbáceas perenes sobre a produção de bananeira cultivar Nanicão.

\section{MATERIALE MÉTODOS}

O experimento foi realizado em condições de campo, em Seropédica, R.J, entre abril de 1999 a julho de 2000, num Argissolo Vermelho - Amarelo distrófico, cujos resultados da análise química de amostras de terra (0-20 $\mathrm{cm})$, segundo Embrapa (1997), apresentaram os seguintes valores: $\mathrm{pH}$ em $\mathrm{H}_{2} \mathrm{O}, 4,4 ; \mathrm{Al}^{+3}, 0,2 \mathrm{cmol} / \mathrm{dm}^{3} ; \mathrm{Ca}^{+2}, 2,5$ $\mathrm{cmol}_{\mathrm{c}} / \mathrm{dm}^{3} ; \mathrm{Mg}^{+2}, 1,4 \mathrm{cmol}_{\mathrm{c}} / \mathrm{dm}^{3} ; \mathrm{K}^{+}, 46 \mathrm{mg} / \mathrm{kg}$ e $\mathrm{P}_{\text {Mehlich } 1}$ $3,0 \mathrm{mg} / \mathrm{kg}$ de solo.

$\mathrm{O}$ delineamento experimental adotado foi o de blocos ao acaso, com cinco tratamentos e quatro repetições. Os tratamentos constaram das espécies perenes que formam a cobertura viva consorciadas à bananeira: amendoim forrageiro (Arachis pintoi Krap. \& Greg), cudzu tropical (Pueraria phaseoloides Benth.), siratro (Macroptilium atropurpureum Urb.), vegetação espontânea (dominada por capim colonião - Panicum maximum Jacq.) e vegetação espontânea $+\mathrm{N}$-fertilizante. Cudzu tropical e siratro possuem crescimento volúvel, enquanto o amendoim forrageiro e capim colonião apresentam crescimento rasteiro e ereto, respectivamente.

As leguminosas herbáceas perenes foram estabelecidas em maio de 1996, usando-se mudas produzidas em bandeja de poliestireno expandido para o amendoim forrageiro e sementes para o cudzu tropical e siratro, na densidade de 20 plantas $/ \mathrm{m}^{2}$. Estirpes de bactérias do gênero Rhizobium, recomendadas pela Embrapa Centro Nacional de Pesquisa de Agrobiologia, foram inoculadas nas mudas e sementes. Já, as bananeiras cultivar nanicão subgrupo Cavendish (AAA), foram implantadas em junho de 1998 no espaçamento de $3 \times 3 \mathrm{~m}$, a partir de mudas inoculadas com fungos micorrízicos arbusculares Glomus clarum (57 esporos/muda) e Gigaspora margarita (18 esporos/muda).

A adubação do primeiro ano de cultivo das bananeiras foi $18 \mathrm{~g}$ de FTE-BR $12,16 \mathrm{~g}$ de $\mathrm{P}_{2} \mathrm{O}_{5}$ (na forma de termofosfato) e $172 \mathrm{~g}$ de $\mathrm{K}_{2} \mathrm{O}$ (na forma de $\mathrm{KCl}$ ) por planta. A partir do segundo ano, as doses de $\mathrm{P}$ e $\mathrm{K}$ foram aumentadas para $47 \mathrm{~g}$ de $\mathrm{P}_{2} \mathrm{O}_{5}$ e $196 \mathrm{~g}$ de $\mathrm{K}_{2} \mathrm{O}$ por planta, enquanto que o FTE-BR 12 foi mantido na mesma quantidade. A aplicação de FTE-BR 12 foi feita em dose única no mês de janeiro, enquanto termofosfato e $\mathrm{KCl}$ eram parcelados em doses idênticas nos meses de janeiro e março. No tratamento vegetação espontânea $+\mathrm{N}$-fertilizante, a dose de nitrogênio foi $36 \mathrm{~g} /$ planta ( $40 \mathrm{~kg} / \mathrm{ha}$ de nitrogênio) no primeiro ano e $81 \mathrm{~g} /$ planta $(90 \mathrm{~kg} / \mathrm{ha}$ de nitrogênio) a partir do segundo ano. O nitrogênio era incorporado 
na forma de uréia, parcelado em doses iguais nos meses de janeiro e março.

As bananeiras eram manejadas no sistema mãe, filha e neta, realizando-se desbrota quando necessário. Já, as coberturas vivas eram roçadas uma vez ao ano, geralmente entre dezembro e janeiro, mantendo-se os resíduos na superfície do solo. O manejo consistia ainda do coroamento e controle de ervas espontâneas, realizado a cada dois meses.

Durante o crescimento das bananeiras da segunda geração (abril de 1999 a julho de 2000), foram feitas medições mensais de altura das plantas e número de folhas emitidas. Por ocasião da colheita dos cachos da segunda geração, as variáveis avaliadas foram: produtividade, peso do cacho, peso das pencas, número de frutos por cacho, número de pencas por cacho, comprimento dos frutos, diâmetro dos frutos e peso dos frutos, nas quatro plantas centrais da parcela. Foi estimada também a porcentagem de cachos colhidos, por meio da proporção de cachos em função da população de plantas por unidade de área.

Os dados foram submetidos à análise de variância, adotando-se teste $\mathrm{F}$ a $5 \%$ de probabilidade, com auxílio do SAEG versão 9.1. Para as variáveis que apresentaram diferença significativa pelo teste F, aplicou-se o teste ScottKnott a 5\% para comparação de médias.

\section{RESULTADOS E DISCUSSÃO}

Pode-se observar na Figura 1, que as coberturas vivas formadas pelas leguminosas proporcionaram maior crescimento das bananeiras, com base na altura, notadamente a partir do $6^{\circ}$ mês. Nota-se, também, que a adubação equivalente a $90 \mathrm{~kg} \mathrm{~N} \mathrm{ha}^{-1}$ na presença de cobertura com o capim colonião (vegetação espontânea) não foi suficiente para promover o crescimento ao nível daquele observado com as coberturas vivas de siratro, amendoim forrageiro e cudzu tropical. Destaca-se que o maior crescimento das plantas na presença das leguminosas denota as vantagens do uso dessas coberturas, quando comparadas com uma espécie como o capim colonião (gramínea). Observa-se ainda, que o cudzu tropical proporcionou, já aos 12 meses, plantas com o dobro da altura daquelas encontradas com a cobertura viva da vegetação espontânea na presença de $\mathrm{N}$-fertilizante.

O número de folhas emitidas das bananeiras foi maior nas fruteiras desenvolvidas no solo coberto com as leguminosas siratro, amendoim forrageiro e cudzu tropical, em relação ao dos tratamentos com vegetação espontânea (com e sem $\mathrm{N}$-fertilizante) (Tabela 1 ). Os resultados relacionados ao crescimento mais rápido das bananeiras consorciadas com as leguminosas cudzu tropical, siratro e amendoim forrageiro, evidenciam o efeito benéfico da

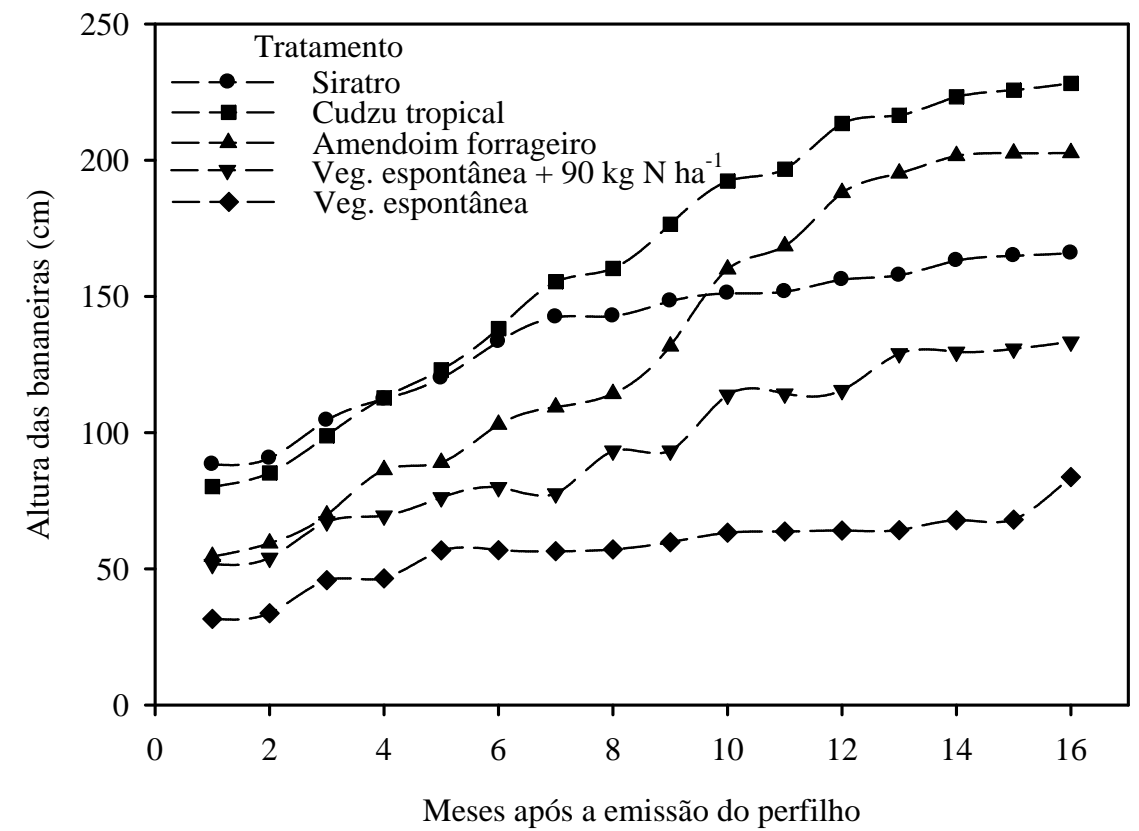

Figura 1 - Altura de bananeiras cultivadas em solo com cobertura viva de leguminosas herbáceas perenes e vegetação espontânea (dominada por capim colonião). Período de abril/1999 a julho/2000. 
cobertura com essas espécies no desenvolvimento vegetativo da bananeira. A partir desses resultados, podese indicar que as leguminosas antecipam o início do período reprodutivo das bananeiras, quando comparadas com as plantas cultivadas na cobertura formada pela vegetação espontânea, mesmo com a aplicação de $\mathrm{N}$-fertilizante. O lançamento do cacho é função do número de folhas emitidas, que de acordo com Borges et al. (1997), se inicia a partir da emissão de 40 folhas.

A proporção de cachos colhidos também foi afetada pelo uso de plantas de cobertura (Tabela 1). Aos 10 meses após a emissão do perfilho, foram colhidos $100 \%$ dos cachos das bananeiras cultivadas em consórcio com cobertura de cudzu tropical, $85 \%$ no consórcio com siratro e $56,25 \%$ no consórcio com amendoim forrageiro. Por outro lado, nesse mesmo período foram colhidos apenas $12,50 \%$ dos cachos das bananeiras consorciadas com vegetação espontânea (dominada pelo capim colonião). Quando as bananeiras foram adubadas com $\mathrm{N}$-fertilizante (equivalente a $90 \mathrm{~kg} \mathrm{~N} \mathrm{ha}^{-1}$ ), a proporção de cachos colhidos aumentou para $31,25 \%$, índice ainda inferior aos observados com a presença das leguminosas. A precocidade da colheita é uma característica desejável, por determinar maior rapidez no retorno dos investimentos realizados.

Em relação aos parâmetros fitotécnicos relacionados à produtividade, o número de pencas e de frutos foram menos influenciados pelas coberturas vivas do que os parâmetros relativos ao peso (Tabela 2), reflexo do menor efeito ambiental, em especial menor competição por recursos hídricos sobre esses parâmetros, também observado para outras espécies vegetais como tremoço-azul, nabo forrageiro e ervilha forrageira quando consorciadas com pomar de pessegueiro (Rufato et al., 2007). Paiva et al. (2007) ressaltam que o sucesso do consórcio entre fruteiras e plantas de cobertura de solo, depende do respeito às características peculiares a cada espécie, no referente as suas necessidades hídricas e nutricionais, caso contrário não haverá adaptação ao sistema ou a concorrência entre plantas será tão grande que comprometerá o seu nível econômico de produtividade.

Contudo, nota-se que tanto o número de pencas como o de frutos, no tratamento controle (vegetação espontânea sem $\mathrm{N}$-fertilizante), foram menores do que nos demais tratamentos, face às condições edafoclimáticas desfavoráveis para o crescimento das plantas, que provocaram impacto marcante no desempenho vegetativo e produtivo das bananeiras (Figura 1 e Tabela 1). Resultados de pesquisa apontaram que a vegetação predominada por capim colonião acarreta em menor umidade do solo (Perin et al., 2002), menor atividade de microrganismos e imobilização de nutrientes, em especial nitrogênio, pela elevada relação $\mathrm{C} / \mathrm{N}$ (Espindola et al., 2006a).

$O$ peso do cacho e da penca foram também positivamente influenciados pelas coberturas vivas formadas pelas leguminosas, quando comparados com a vegetação espontânea na ausência de $\mathrm{N}$-fertilizante nas bananeiras, exceto com a cobertura de amendoim forrageiro (Tabela 2). Condições edafoclimáticas desfavoráveis podem, no entanto, ocasionar reduções no peso de cachos, peso das pencas e na produtividade de bananeiras consorciadas com leguminosas herbáceas perenes, como é relatado para essa fruteira cultivada em solo coberto com amendoim forrageiro. Uma das possíveis causas desses resultados é a competição por água entre a leguminosa e a bananeira (Johns, 1994). Trabalhos recentes mostraram que

Tabela 1 - Número total de folhas emitidas e proporção de cachos colhidos aos 10 meses após emissão do perfilho de bananeiras cultivar Nanicão, consorciadas com coberturas vivas de leguminosas herbáceas perenes ou com vegetação espontânea com e sem N-fertilizante.

\begin{tabular}{|c|c|c|}
\hline \multirow[t]{2}{*}{ Tratamento } & Total de folhas emitidas & Cachos colhidos \\
\hline & ----------- $\left(\mathrm{N}^{\mathrm{o}}\right)$----------- & ---------- $(\%)$----------- \\
\hline Siratro & $36,50 \mathrm{~A}^{2}$ & $85,00 \mathrm{~B}$ \\
\hline Cudzu tropical & $33,02 \mathrm{~A}$ & $100,00 \mathrm{~A}$ \\
\hline Amendoim forrageiro & $35,96 \mathrm{~A}$ & $56,25 \mathrm{C}$ \\
\hline Veg. espont. ${ }^{1}+\mathrm{N}$ & $28,63 \mathrm{~B}$ & $31,25 \mathrm{D}$ \\
\hline Veg. espontânea & $23,56 \mathrm{~B}$ & $12,50 \mathrm{E}$ \\
\hline $\mathrm{CV}(\%)$ & 16,21 & 20,31 \\
\hline
\end{tabular}

\footnotetext{
${ }^{1}$ Vegetação espontânea: predomínio da gramínea capim colonião (Panicum maximum)

${ }^{2}$ Médias seguidas da mesma letra dentro da coluna, não diferem entre si pelo teste Scott-Knott $(\mathrm{p}>0,05)$.
} 
Tabela 2 - Número de cachos, peso do cacho e das pencas, número de frutos e de pencas por cacho, e produtividade de bananeiras cultivar Nanicão, consorciadas com coberturas vivas de leguminosas herbáceas perenes ou com vegetação espontânea com e sem $\mathrm{N}$-fertilizante.

\begin{tabular}{|c|c|c|c|c|c|c|}
\hline \multirow{4}{*}{$\begin{array}{c}\text { Cobertura } \\
\text { Viva }\end{array}$} & \multicolumn{6}{|c|}{ Produção de frutos } \\
\hline & \multirow{3}{*}{$\begin{array}{c}\text { Cacho } \\
\text { colhido } \\
\left(\mathrm{n}^{\mathrm{o}} / \mathrm{ha}\right)\end{array}$} & \multirow{3}{*}{$\begin{array}{l}\text { Cacho } \\
\text { inteiro } \\
--\mathrm{kg}--\end{array}$} & \multicolumn{2}{|c|}{ Penca } & \multirow{3}{*}{$\begin{array}{c}\text { Fruto } \\
\text { (n\%/cacho) }\end{array}$} & \multirow{3}{*}{$\begin{array}{l}\text { Produtividade } \\
---\mathrm{Mg} \mathrm{ha}^{-1} \text {-- }\end{array}$} \\
\hline & & & 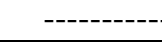 & ----------- & & \\
\hline & & & (n\%cacho) & (kg/cacho) & & \\
\hline Siratro & 944 & $11,51 a^{2}$ & $6,92 \mathrm{a}$ & $10,58 \mathrm{a}$ & $95,13 \mathrm{a}$ & $10,87 \mathrm{a}$ \\
\hline Cudzu tropical & 1.111 & $10,89 \mathrm{a}$ & $6,44 \mathrm{a}$ & $10,16 \mathrm{a}$ & 89,33 a & $12,09 \mathrm{a}$ \\
\hline Amend. forrageiro & 625 & $8,49 \mathrm{~b}$ & $5,88 \mathrm{a}$ & $7,99 \mathrm{~b}$ & $77,50 \mathrm{a}$ & $5,31 \mathrm{~b}$ \\
\hline Veg. espont. ${ }^{1}+\mathrm{N}$ & 347 & $11,54 \mathrm{a}$ & $6,42 \mathrm{a}$ & $10,92 \mathrm{a}$ & $78,00 \mathrm{a}$ & $4,01 \mathrm{~b}$ \\
\hline Veg. espontânea & 139 & $6,27 \mathrm{~b}$ & $4,25 \mathrm{~b}$ & $5,95 \mathrm{~b}$ & $39,75 \mathrm{~b}$ & $1,04 \mathrm{c}$ \\
\hline CV (\%) & - & 20 & 13 & 20 & 16 & 18 \\
\hline
\end{tabular}

${ }^{1}$ Vegetação espontânea: predomínio da gramínea capim colonião (Panicum maximum)

${ }^{2}$ Médias seguidas da mesma letra dentro da coluna, não diferem entre si pelo teste Scott-Knott $(\mathrm{p}>0,05)$.

o amendoim forrageiro apresenta alta produção de raízes, principalmente nas camadas superficiais, e provoca redução expressiva nos níveis de umidade do solo, quando comparado ao cultivo de cudzu tropical e siratro (Perin, 2001; Perin et al., 2002). Trabalhos de pesquisa também mostraram que o amendoim forrageiro forma excelente cobertura de solo, apresenta alto potencial de produção de biomassa e capacidade de estoque de nutrientes (Perin et al., 2003), e maior velocidade de decomposição dos resíduos de parte aérea (Espindola et al., 2006a), quando comparado com siratro e cudzu tropical. Porém, esses atributos não devem ser os únicos considerados na tomada de decisão sobre a combinação mais adequada entre espécie de leguminosa para formação de cobertura viva para consorciação com fruteiras, principalmente em regiões com déficit hídrico pronunciado.

A associação das características de precocidade (Tabela 1) e atributos relacionados à produção de frutos das bananeiras na presença das coberturas de siratro e cudzu tropical (Tabela 2) resultou ganhos de produtividade superiores em, aproximadamente 10 vezes aos obtidos com a vegetação espontânea. Nota-se que, embora a adubação nitrogenada mineral tenha proporcionado aumento na produtividade das plantas, em relação ao controle, o benefício foi menor do que o observado com o consórcio com siratro e cudzu tropical. Espindola et al. (2006b) demonstraram que a fixação biológica de nitrogênio pelo siratro e cudzu tropical alcançou, respectivamente, 89 e $305 \mathrm{~kg}$ de $\mathrm{N} \mathrm{ha}^{-1}$, contribuindo com até $40 \%$ do $\mathrm{N}$ presente nas bananeiras, por ocasião do $1^{\circ}$ ciclo de colheita. Dessa forma, a cobertura permanente do solo com essas leguminosas promove condições adequadas ao desenvolvimento das plantas, acarretando ganhos de produtividade e eliminação da adubação nitrogenada no bananal.

Do ponto de vista do manejo da cobertura viva com as leguminosas, após o estabelecimento, a roçada não é uma prática imprescindível, todavia, permite aumentar a quantidade de matéria orgânica depositada sobre o solo, visto que essas plantas suportam cortes, rebrotando com facilidade (Perin, 2001). A roçada, além de funcionar como uma adubação verde auxilia na orientação da própria cobertura viva. Particularmente, siratro e cudzu apresentam queda natural de folhas, o que proporciona a deposição natural e contínua de matéria orgânica, porém, o mesmo não ocorre com o amendoim forrageiro, que requer roçada para potencializar o benefício da adubação verde. No bananal, é recomendável o coroamento, principalmente se a cobertura é formada pelo siratro ou cudzu tropical, que têm hábitos volúveis; assim, a cobertura viva não prejudicará nem o perfilhamento das plantas, e nem os tratos culturais requeridos pela cultura.

\section{CONCLUSÕES}

Nas condições de manejo empregadas, as coberturas de solo com siratro e cudzu tropical conferiram crescimento e produtividade de banana maior do que a cobertura viva formada pelo amendoim forrageiro e pela vegetação espontânea, dominada pelo capim colonião. 
O potencial benéfico das leguminosas cudzu tropical e siratro como coberturas vivas capazes de proporcionar aumentos na produtividade de banana, qualifica essas espécies como alternativas promissoras para a fertilização do solo, adubação e nutrição das plantas de bananeira.

\section{REFERÊNCIAS BIBLIOGRÁFICAS}

BORGES, A.L.; OLIVEIRA, A.M.G.; SOUZA, L.S. Solos, nutrição e adubação. In: ALVES, E.J. (Org.). A cultura da banana: aspectos técnicos, socioeconômicos e agroindustriais. Brasília: EMBRAPA-SPI/EMBRAPACNPMF, 1997. p.197-260.

CANELLAS, L.P.; ESPINDOLA, J.A.A.; GUERRA, J.G.M.; TEIXEIRA, M.G.; VELLOSO, A.C.X.; RUMJANEK, V.M. Phosphorus analysis in soil under herbaceous perennial leguminous cover by nuclear magnetic spectroscopy. Pesquisa Agropecuária Brasileira, Brasília, v.39, p.589-596, 2004.

DERPSCH, R.; ROTH, C.H.; SIDIRAS, N.; KOPKE, U. Controle da erosão no Paraná, Brasil: sistemas de cobertura do solo, plantio direto e preparo conservacionista do solo. Londrina: GTZ-IAPAR, 1991. $272 p$.

DUDA, G.P.; GUERRA, J.G.M.; MONTEIRO, M.T.; DEPOLLI, H.; TEIXEIRA, M.G. Perennial herbaceuous legumes as live soil mulches and their effects on $\mathrm{C}, \mathrm{N}$ and $\mathrm{P}$ of the microbial biomass. Scientia Agrícola, Piracicaba, v.60, n.1, p.139-147, 2003.

EMPRESA BRASILERIA DE PESQUISA AGROPECUÁRIA. Centro Nacional de Pesquisa de Solos. Manual de métodos de análise de solo. Rio de Janeiro, 1997. 212p.

ESPINDOLA, J.A.A.; GUERRA, J.G.M.; ALMEIDA, D.L.; TEIXEIRA, M.G.; URQUIAGA, S. Decomposição e liberação de nutrientes acumulados em leguminosas herbáceas perenes consorciadas com bananeira. Revista Brasileira de Ciência do Solo, Viçosa, v.30, n.2, p.321328, 2006a.

ESPINDOLA, J.A.A.; GUERRA, J.G.M.; PERIN, A.; TEIXEIRA, M.G.; ALMEIDA, D.L.; URQUIAGA, S.; BUSQUET, R.B. Bananeiras consorciadas com leguminosas herbáceas perenes utilizadas como coberturas vivas. Pesquisa Agropecuária Brasileira, Brasília, v.41, n.3, p.415-420, 2006b.

ESPINDOLA, J.A.A.; OLIVEIRA, S.J.C.R. de; CARVALHO, G.J.A. de; SOUZA, C.L.M. de; PERIN, A.; GUERRA, J.G.M.; TEIXEIRA, M.G. Potencial alelopático e controle de plantas invasoras por leguminosas herbáceas perenes consorciadas com bananeira. Seropédica: Embrapa Agrobiologia, 2000. 8p. (Comunicado técnico, 47).

\section{INSTITUTO BRASILEIRO DE GEOGRAFIA E} ESTATÍSTICA. Anuário estatístico do Brasil. Rio de Janeiro, 2002. 25p.

JOHNS, G.G. Effect of Arachis pintoi groundcover on performance of bananas in northern New South Wales. Australian Journal of Experimental Agriculture, Victoria, v.34, p.1197-1204, 1994.

MEDINA, J.C.; BLEINROTH, E.W.; MARTIN, Z.J. de; TRAVAGLINI, D.A.; OKADA, M.; QUAST, D.G.; HASHIZUME, T.; MORETTI, V.A.; BICUDO NETO, L.C.; ALMEIDA, L.A.S.B.; RENESTO, O.V. Banana: cultura, matéria-prima, processamento e aspectos econômicos. 2.ed. Campinas: Instituto de Tecnologia de Alimentos, 1995. 302p. (Frutas Tropicais, 3).

PAIVA, J.R.; SANTOS, F.J.S.; CACAU, J.; SOUZA, R.N.M.; SOBRAL, A.R.A. Policultivo com diferentes espécies frutíferas. Ciência e Agrotecnologia, Lavras, v.30, n.1, p.81-87, jan./fev. 2007.

PERIN, A. Desempenho de leguminosas herbáceas perenes com potencial de utilização para cobertura viva e seus efeitos sobre alguns atributos físicos do solo. 2001. 144f. Dissertação (Mestrado em Ciência do Solo)Universidade Federal Rural do Rio de Janeiro, Seropédica, 2001.

PERIN, A.; GUERRA, J.G.M.; TEIXEIRA, M.G. Cobertura do solo e acumulação de nutrientes pelo amendoim forrageiro. Pesquisa Agropecuária Brasileira, Brasília, v.38, n.7, p.791-796, jul. 2003.

PERIN, A.; GUERRA, J.G.M.; TEIXEIRA, M.G.; PEREIRA, M.G.; FONTANA, A. Efeito da cobertura viva com leguminosas herbáceas perenes na agregação de um argissolo. Revista Brasileira de Ciência do Solo, Campinas, v.26, p.713-720, 2002. 
RUFATO, L.; RUFATO, A.R.; KRETZSCHMAR, A.A.; PICOLOTTO, L.; FACHINELLO, J.C. Coberturas

vegetais no desenvolvimento vegetativo de plantas de pessegueiro. Revista Brasileira de Fruticultura,

Jaboticabal, v.29, n.1, p.107-109, abr. 2007.
WILES, L.J.; WILLIAM, R.D.; CRABTREE, G.D.;

RADOSEVICH, S.R. Analyzing competition between a living mulch and a vegetable crop in an interplanting system. Journal of the American Society for Horticulture Science Journal, Alexandria, v.114, p.1029-1034, 1989. 